\title{
Asymptotic singularities of planar parallel 3-RPR manipulators
}

\author{
Michel Coste
}

\begin{abstract}
We study the limits of singularities of planar parallel 3-RPR manipulators as the lengths of their legs tend to infinity, paying special attention to the presence of cusps. These asymptotic singularities govern the kinematic behavior of the manipulator in a rather large portion of its workspace.
\end{abstract}

Key words: parallel robots, singularities.

\section{Introduction}

Planar 3-RPR manipulators have been extensively studied, see for instance [1-10]. Much attention has been paid to the analysis of the singularities of these manipulators. These singularities govern the kinematic properties of the manipulators, for instance their ability to perform singularity-free assembly mode changing.

It has been reported in several papers that the picture of the singularities in the actuated joint space stabilizes when the lengths of the legs are sufficiently large. Actually, in several examples, this stabilization appears already when the lengths of legs are two or three times the lengths of the sides of the base and platform triangles. See for instance Figure 1 extracted from the paper [8].

We study in this paper this stable configuration, introducing the limits of singularities of planar parallel 3-RPR manipulators as the lengths of their legs tends to infinity. These asymptotic singularities govern the kinematic behavior of the manipulator in a rather large portion of its workspace. We classify the 3-RPR manipulators with respect to the features of this stable configuration of singularities. We pay special attention to the presence of cusps in the singular locus, since their presence is crucial for the possility of singularity-free assembly mode changing.

Michel Coste

IRMAR, Université de Rennes 1 - CNRS

e-mail: michel.coste@univ-rennes1.fr 


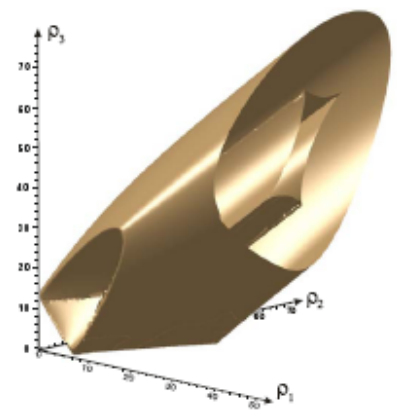

Fig. 1 Asymptotic stability of the section of the singularity surface of a 3-RPR manipulator

\section{Modelization}

The notations, the parameters and the variables we use are explained in Figure 2.

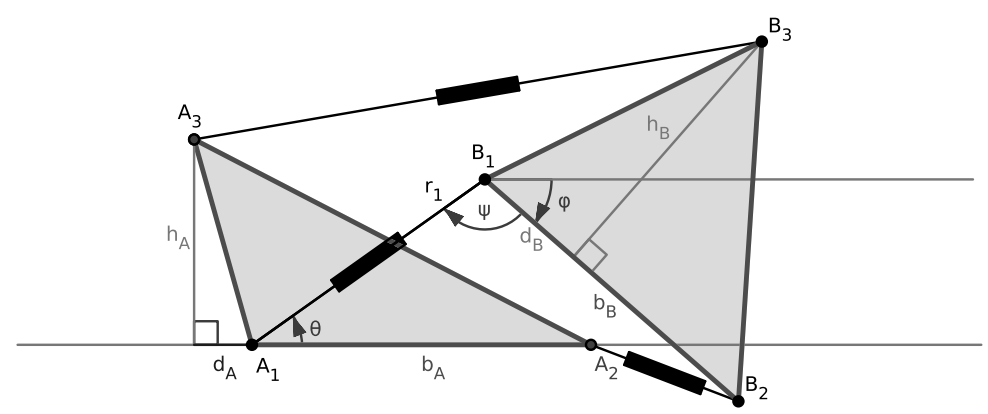

Fig. 2 3-RPR, parameters and variables used

The geometry of the base (resp. platform) is described by the parameters $b_{A}, h_{A}, d_{A}$ (resp. $b_{B}, h_{B}, d_{B}$ and a $\operatorname{sign} \varepsilon= \pm 1$ ). In the direct orthonormal frame attached to the base with origin $A_{1}, A_{2}$ has coordinates $\left(b_{A}, 0\right)\left(b_{A}>0\right)$ and $A_{3}$ has coordinates $\left(d_{A}, h_{A}\right)\left(h_{A}>0\right)$. In the direct orthonormal frame attached to the platform with origin $B_{1}, B_{2}$ has coordinates $\left(b_{B}, 0\right)\left(b_{B}>0\right)$ and $B_{3}$ has coordinates $\left(d_{B}, \varepsilon h_{B}\right)$ $\left(h_{B}>0\right)$; the sign $\varepsilon$ is -1 when the triangle $B_{1} B_{2} B_{3}$ is oriented clockwise. We always assume that neither triangle is flat, i.e. $b_{A} h_{A} b_{B} h_{B} \neq 0$. The position of the vertex $B_{1}$ of the platform is given in polar coordinates by the angle $\theta$ and the length $r_{1}$ of the leg $A_{1} B_{1}$. The orientation of the platform is given by the angle $\varphi$.

We shall also use the angle $\psi=\theta-\varphi+\pi$ instead of $\varphi$ in order to simplify some equations. 
We denote by $r_{2}$ and $r_{3}$ the lengths of the legs $A_{2} B_{2}$ and $A_{3} B_{3}$. These lengths are given by

$$
\begin{aligned}
r_{2}^{2} & =r_{1}^{2} \\
& +2 r_{1}\left(\left(b_{B} \cos \varphi-b_{A}\right) \cos \theta+b_{B} \sin \varphi \sin \theta\right)-2 b_{A} b_{B} \cos \varphi+b_{A}^{2}+b_{B}^{2} \\
r_{3}^{2} & =r_{1}^{2} \\
& +2 r_{1}\left(\left(d_{B} \cos \varphi-\varepsilon h_{B} \sin \varphi-d_{A}\right) \cos \theta+\left(d_{B} \sin \varphi+\varepsilon h_{B} \cos \varphi-h_{A}\right) \sin \theta\right) \\
& -2\left(d_{A} d_{B}+\varepsilon h_{A} h_{B}\right) \cos \varphi+2\left(\varepsilon d_{A} h_{B}-h_{A} d_{B}\right) \sin \varphi+d_{A}^{2}+d_{B}^{2}+h_{A}^{2}+h_{B}^{2}
\end{aligned}
$$

\section{Asymptotic direct kinematic problem and singularities}

We are interested in the limits of $r_{1}-r_{2}$ and $r_{1}-r_{3}$ as $r_{1}$ tends to infinity. We denote these limits by $\ell_{2}$ and $\ell_{3}$, repectively. They are easily obtained from Equations (1) as

$$
\begin{aligned}
& \ell_{2}=\left(b_{A}-b_{B} \cos \varphi\right) \cos \theta-b_{B} \sin \varphi \sin \theta \\
& \ell_{3}=\left(d_{A}+\varepsilon h_{B} \sin \varphi-d_{B} \cos \varphi\right) \cos \theta+\left(h_{A}-d_{B} \sin \varphi-\varepsilon h_{B} \cos \varphi\right) \sin \theta
\end{aligned}
$$

Equations (2) are to be seen as describing the asymptotic inverse kinematic mapping (IKM). The asymptotic direct kinematic problem (DKP) is solving Equations (2) for $\theta$ and $\varphi$. This can be done by writing Equations (2) in terms of $u=\tan (\varphi / 2)$, eliminating $u$ and writing the resultant obtained in $t=\tan (\theta / 2)$; this process gives the following quartic characteristic equation in $t$ :

$$
\begin{aligned}
0= & \left(\left(d_{B} l_{2}-b_{B} l_{3}\right)\left(t^{2}+1\right)+2 h_{A} b_{B} t+\left(b_{A} d_{B}-d_{A} b_{B}\right)\left(t^{2}-1\right)\right)^{2} \\
& +h_{B}^{2}\left(\left(l_{2}\left(t^{2}+1\right)+b_{A}\left(t^{2}-1\right)\right)^{2}-b_{B}^{2}\left(t^{2}+1\right)^{2}\right)
\end{aligned}
$$

If we have a value of $\theta$ such that $t=\tan (\theta / 2)$ is a solution of Equation (3), carrying this value in Equations (2) yields a linear system in $\cos \varphi$ and $\sin \varphi$, whose determinant $-\varepsilon b_{B} h_{B}$ does not vanish. So we obtain a unique solution $(\theta, \varphi)$ to the symptotic DKP.

The asymptotic DKP has degree 4 and has no more than 4 solutions.

The Jacobian determinant of the asymptotic IKM (2) is

$$
\begin{aligned}
\mathrm{Jac}= & \left(h_{A} b_{B} \cos \theta^{2}+\left(b_{A} d_{B}-d_{A} b_{B}\right) \cos \theta \sin \theta+\varepsilon b_{A} h_{B} \sin \theta^{2}\right) \sin \varphi \\
& +\left(\left(\varepsilon b_{A} h_{B}-h_{A} b_{B}\right) \cos \theta \sin \theta+\left(d_{A} b_{B}-b_{A} d_{B}\right) \sin \theta^{2}\right) \cos \varphi
\end{aligned}
$$

The equation $\mathrm{Jac}=0$ can easily be solved in $\tan (\varphi)$ :

$$
\tan (\varphi)=\frac{\left(h_{A} b_{B}-\varepsilon b_{A} h_{B}\right) \tan (\theta)+\left(b_{A} d_{B}-d_{A} b_{B}\right) \tan (\theta)^{2}}{h_{A} b_{B}+\left(b_{A} d_{B}-d_{A} b_{B}\right) \tan (\theta)+\varepsilon b_{A} h_{B} \tan (\theta)^{2}}
$$


There is no surprise that we get in this way the curve of poles of the rational parameterization $r_{1}^{\text {Sing }}(\varphi, \theta)$ of the singularity surface in the workspace obtained in [10]. This singularity curve in the torus of variables $(\varphi, \theta)$ was already studied in the quoted paper, where it is proved that it has two branches (connected components), one passing through the point $\varphi=0, \theta=0$ and the other passing through $\varphi=\pi, \theta=$ 0 obtained from the first by a translation of $\pi$ on the angular variable $\varphi$.

The image by the asymptotic IKM of the singularity curve in the plane of variables $\left(\ell_{2}, \ell_{3}\right)$ consists also of two branches. This curve is generically an irreducible curve of degree 8 , not rational. However, it is possible to obtain parameterizations by analytic functions of $\theta$ of each of the branches by substituting in Equations (2) the expressions for the trigonometric fonction of $\varphi$ extracted from Equation (5). One has to be careful in the determination of the angle $\varphi$ from its tangent, in order to ensure the continuity. One obtains for the first branch (the inner branch)

$$
\begin{aligned}
& \ell_{2}^{\text {in }}=\frac{\left(b_{A} \sqrt{\delta}-h_{A} b_{B}^{2}\right) \cos \theta-b_{B}\left(b_{A} d_{B}-d_{A} b_{B}\right) \sin \theta}{\sqrt{\delta}} \\
& \ell_{3}^{\text {in }}=\frac{\left(d_{A} \sqrt{\delta}-h_{A} b_{B} d_{B}\right) \cos \theta+\left(h_{A} \sqrt{\delta}-d_{B}\left(b_{A} d_{B}-d_{A} b_{B}\right)-b_{A} h_{B}^{2}\right) \sin \theta}{\sqrt{\delta}}
\end{aligned}
$$

where $\delta=\left(h_{A} b_{B} \cos \theta+\left(b_{A} d_{B}-d_{A} b_{B}\right) \sin \theta\right)^{2}+b_{A}^{2} h_{B}^{2} \sin \theta^{2}$. Note that $\delta$ is always $>0$, which ensures the analyticity in $\theta$ of the formulas (6). For the second branch (the outer branch), one simply replaces all occurences of $\sqrt{\delta}$ by $-\sqrt{\delta}$ in formulas (6).

\section{Examples}

We show here a few examples of asymptotic image singularity curves in the plane $\left(\ell_{2}, \ell_{3}\right)$. The curves are drawn using formulas (6) and their variants for the outer branch.

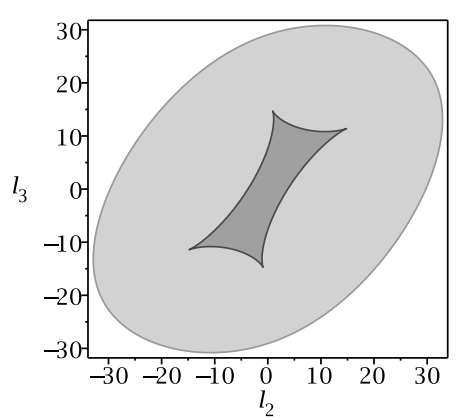

Fig. 3 The asymptotic image singularity curve for the Innocenti-Merlet manipulator (diamond) 
The first example (Figure 3) is the Innocenti-Merlet manipulator [7], with $b_{A}=$ 15.9, $h_{A}=10, d_{A}=0, b_{B}=17, \varepsilon h_{B}=16.1, d_{B}=13.2$; this is also the example of Figure 1, and one recovers the stable section of the singularity surface for large lengths of legs. The light gray indicates two solutions for the DKP, the dark gray four solutions. The inner branch has four cusps and no double point; we call this configuration a diamond.
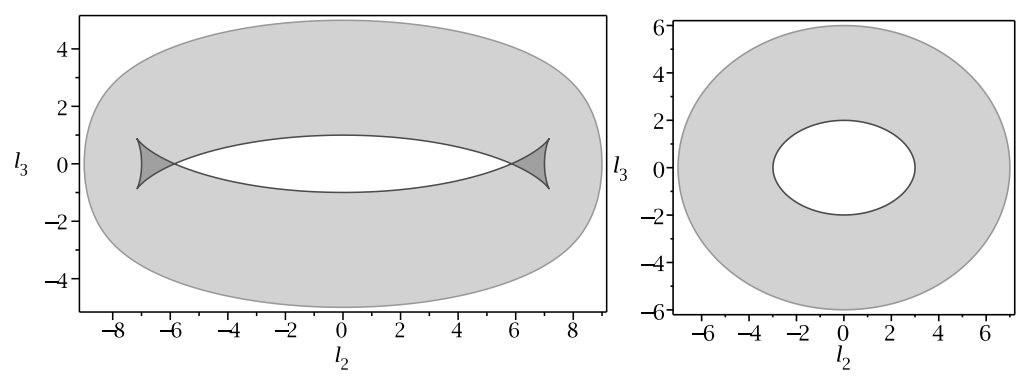

Fig. 4 Other configurations for the asymptotic image singularity curve (swallowtail and annulus)

The next examples (Figure 4) show two other configurations for the image singularity curves, with the same gray scale code. In both examples we have $d_{A}=d_{B}=0$. The example on the left is for $b_{A}=8, h_{A}=3, b_{B}=1, \varepsilon h_{B}=2$. The inner branch in this case has four cusps and two double points; we call this configaration a swallowtail. The example on the right is for $b_{A}=5, h_{A}=4, b_{B}=2, \varepsilon h_{B}=2$. Here the inner branch is just an oval; we call this configuration an annulus.

We shall explain in the next section why these three examples show all possible stable configurations for the asymtotic image singularity curve: diamond, swallowtail or annulus.

\section{Asymptotic kinematics and wavefronts of an ellipse}

The equations 2 for the asymptotic IKM take a more symmetric form when they are written, after substituting $\varphi$ with $\theta-\psi+\pi$, as:

$$
\left(\begin{array}{l}
\ell_{2} \\
\ell_{3}
\end{array}\right)=\left(\begin{array}{cc}
b_{A} & 0 \\
d_{A} & h_{A}
\end{array}\right)\left(\begin{array}{c}
\cos \theta \\
\sin \theta
\end{array}\right)+\left(\begin{array}{cc}
b_{B} & 0 \\
d_{B} & \varepsilon h_{B}
\end{array}\right)\left(\begin{array}{c}
\cos \psi \\
\sin \psi
\end{array}\right) .
$$

The two summands of the right handside of Equation (7) are parameterizations of ellipses $\mathscr{E}_{A}(\theta)$ and $\mathscr{E}_{B}(\psi)$. By a linear change of coordinates, we can assume that one of the ellipses (say $\mathscr{E}_{B}$ ) is a circle of radius $\rho$. We are then in the situation of the paradigmatic example for wavefronts $[11,12]$ : the wavefront of an ellipse, which is the envelope of the family of circles with radius $\rho$ centered in a point of the ellipse (the image of $\mathscr{E}_{A}$ under the coordinate change). The outer branch of the 
wavefront is always a smooth convex curve, whereas the shape of the inner branch of the wavefront bifurcates at values $\rho=\beta^{2} / \alpha, \beta, \alpha, \alpha^{2} / \beta$ (where $\alpha$ and $\beta$ are respectively the semi-major and semi-minor axes lengths of the ellipse). The stable configurations of the wavefront outside of the bifurcation values are the ones we encountered in the preceding section: diamond, swallowtail and annulus.

There is a cusp of the inner branch of the wavefront, where it is tangent to the circle of radius $\rho$ centered at $P$ on the ellipse, when $\rho$ is equal to the curvature radius of the ellipse at $P$. Hence, there is no cusp on the inner branch of the wavefront when $\rho$ is below the minimum or above the maximum of the curvature radius along the ellipse, which are respectively $\beta^{2} / \alpha$ and $\alpha^{2} / \beta$. These values correspond to the bifurcation between annulus and swallowtail, as shown in Figure 5 (ellipse in dotted line, wavefront in solid line, circles in light gray).
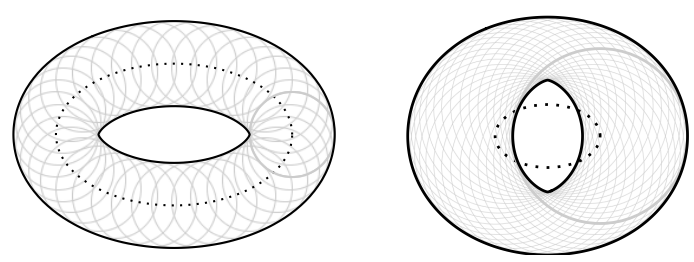

Fig. 5 Bifurcation between annulus and swallowtail for $\rho=\beta^{2} / \alpha$ and $\rho=\alpha^{2} / \beta$

The inner branch of the wavefront has four cusps and no double points for values of $\rho$ between $\beta$ and $\alpha$. These values correspond to the bifurcation between diamond and swallowtail, as shown in Figure 6.
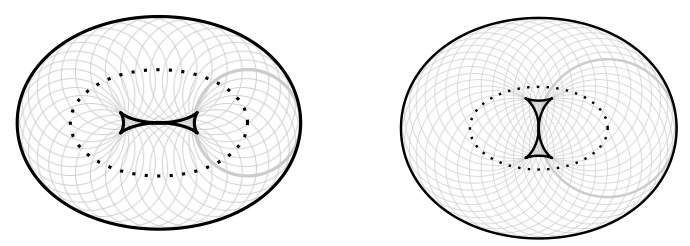

Fig. 6 Bifurcation between diamond and swallowtail for $\rho=\beta$ and $\rho=\alpha$

In conclusion, we know what are the stable configurations (diamond, swallowtail, annulus) for the asymptotic DKP and we can characterize the bifurcations between these stable configurations. We shall make precise the classification of 3-RPR manipulators according to these stable configurations in the following section. 


\section{Classification of 3-RPR manipulators}

We use the analysis of the bifurcation of the wavefront of an ellipse recalled in the preceding section. In order to do that, we change coordinates in the $\left(\ell_{2}, \ell_{3}\right)$-plane so that the ellipse $\mathscr{E}_{B}$ of Equation 7 is a circle of radius 1 in the new coordinates. We set

$$
M_{A}=\left(\begin{array}{cc}
b_{A} & 0 \\
d_{A} & h_{A}
\end{array}\right) \quad M_{B}=\left(\begin{array}{cc}
b_{B} & 0 \\
d_{B} & \varepsilon h_{B}
\end{array}\right) .
$$

The new coordinates $(u, v)$ are given by $\left(\begin{array}{l}u \\ v\end{array}\right)=M_{B}^{-1}\left(\begin{array}{l}\ell_{1} \\ \ell_{2}\end{array}\right)$ and the equation of the ellipse $\mathscr{E}_{A}$ in variables $(u, v)$ is

$$
(u v)^{t}\left(M_{A}^{-1} M_{B}\right) M_{A}^{-1} M_{B}\left(\begin{array}{l}
u \\
v
\end{array}\right)=1
$$

We denote by $\alpha$ and $\beta$ the semi-major and semi-minor axes lengths of the ellipse described by Equation 9. The eigenvalues of the positive symmetric matrix ${ }^{t}\left(M_{A}^{-1} M_{B}\right) M_{A}^{-1} M_{B}$ are $1 / \alpha^{2}$ and $1 / \beta^{2}$, whence we derive an equation whose roots are $\alpha^{2}$ and $\beta^{2}$ :

$$
S_{B}^{2} \lambda^{2}-U_{A, B} \lambda+S_{A}^{2}=0
$$

with

$$
\begin{aligned}
S_{A} & \left.=b_{A} h_{A} / 2, \quad S_{B}=b_{B} h_{B} / 2 \quad \text { (the areas of the triangles }\right), \\
U_{A, B} & =\frac{1}{4}\left(b_{A}^{2} h_{B}^{2}+h_{A}^{2} b_{B}^{2}+\left(b_{A} d_{B}-d_{A} b_{B}\right)^{2}\right) \\
& =\frac{1}{8}\left(a_{A}^{2}\left(b_{B}^{2}+c_{B}^{2}-a_{B}^{2}\right)+b_{A}^{2}\left(c_{B}^{2}+a_{B}^{2}-b_{B}^{2}\right)+c_{A}^{2}\left(a_{B}^{2}+b_{B}^{2}-c_{B}^{2}\right)\right),
\end{aligned}
$$

where $a_{A}, c_{A}, a_{B}, c_{B}$ denote the lengths of the sides $A_{3} A_{1}, A_{2} A_{3}, B_{3} B_{1}, B_{2} B_{3}$ respectively. The second expression for $U_{A, B}$ makes clear that it is symmetric w.r.t. the three sides of the triangles. The inequality $U_{A, B} \geq 2 S_{A} S_{B}$, expressing the fact that the discriminant of Equation (10) is always nonnegative, is known in geometry as the Neuberg-Pedoe inequality $[13,14]$; the equality case is precisely when the two triangles are similar.

The diamond configuration occurs when $\beta<1<\alpha$, i.e. when $\left(1-\alpha^{2}\right)(1-$ $\left.\beta^{2}\right)<0$. The annulus configuration occurs when $1<\beta^{2} / \alpha$ or $1>\alpha^{2} / \beta$, i.e. when $\left(1-\alpha^{4} / \beta^{2}\right)\left(1-\beta^{4} / \alpha^{2}\right)>0$. We transform these inequalities using Equation (10) and obtain the following classification result.

The configuration of the asymptotic singularities of a 3-RPR manipulator is

- a diamond when $U_{A, B}>S_{A}^{2}+S_{B}^{2}$,

- an annulus when $U_{A, B}^{3}<S_{A}^{2} S_{B}^{2}\left(3 U_{A, B}+S_{A}^{2}+S_{B}^{2}\right)$,

- a swallowtail when $U_{A, B}<S_{A}^{2}+S_{B}^{2}$ and $U_{A, B}^{3}>S_{A}^{2} S_{B}^{2}\left(3 U_{A, B}+S_{A}^{2}+S_{B}^{2}\right)$. 
The inequality $U_{A, B} \geq S_{A}^{2}+S_{B}^{2}$ has a geometric interpretation [14]: it is satisfied if and only if there is a pose of the manipulator such that the three legs $A_{1} B_{1}, A_{2} B_{2}$ and $A_{3} B_{3}$ are parallel. No such geometric interpretation seems to be known for the inequality $U_{A, B}^{3}<S_{A}^{2} S_{B}^{2}\left(3 U_{A, B}+S_{A}^{2}+S_{B}^{2}\right)$.

The three stable configurations we have found (diamond, annulus and swallowtail) only have the stable singularities [11] of a projection of a surface to a plane: folds, cusps and transversal intersection of folds. Hence these configurations of asymptotic singularities remain the same for the sections at large values of $r_{1}$ (or $r_{2}$, or $r_{3}$ ) of the singularity surface in the actuated joint space; the precise meaning of "large" depends, of course, on the geometry of the manipulator.

There are non-generic manipulators for which the asymptotic singularities do not belong to a stable configuration. This is the case for the "symmetric" manipulators [15]: in this case the inner branch of the asymptotic singularity curve is reduced to a point. We plan to study the stable perturbations of such manipulators in a future work.

\section{Conclusion}

We studied the asymptotic DKP for 3-RPR manipulators. We have shown that this is a fourth-degree problem. We have established that there are only three possible stable configurations for the asymptotic singularities (diamond, swallowtail and annulus), which remain unchanged for sufficiently large values of the lengths of the legs. We also gave an explicit classification of 3-RPR manipulators with respect to their asymptotic singularities.

\section{References}

1. K.H. HunT, "Structural kinematics of in-parallel actuated robot arms", J. of Mechanisms, Trans. and Aut. in Design 105(4): 705-712, 1983.

2. C. Innocenti and V. Parenti-Castelli, "Singularity-free Evolution From One Configuration to Another in Serial and Fully-Parallel Manipulators", Proc. ASME Design Technical Conferences, DE-Vol. 45, Spatial mechanisms and mechanical systems, pp 553-560, 1992.

3. C.M. GosSELIN and J-P. MERLET, "On the direct kinematics of planar parallel manipulators: special architectures and number of solutions”, Mechanism and Machine Theory, 29(8): 10831097, 1994.

4. P. Wenger and D. Chablat, "Workspace and assembly-modes in fully-parallel manipulators: a descriptive study", Advances in Robot Kinematics and Computational Geometry, Kluwer Academic Publishers, pp. 117-126, 1998.

5. P.R. MCAREE and R.W. DANIEL, "An explanation of never-special assembly changing motions for 3-3 parallel manipulators", The International Journal of Robotics Research, 18(6): 556-574, 1999.

6. I. Bonev, D. Zlatanov, and C. Gosselin, "Singularity Analysis of 3-DOF Planar Parallel Mechanisms via Screw Theory”, ASME Journal of Mechanical Design, 125(3): 573-581, 2003. 
7. J-P. MERlet, Parallel Robots, Springer 2006.

8. M. Zein, P. Wenger, D. Chablat, "Singular Curves in the Joint Space and Cusp Points of 3-RPR parallel manipulators", Robotica, 25(6), 717-724, 2007

9. M.L. Husty, "Non-singular assembly mode change in 3-RPR-parallel manipulators". In Computational Kinematics: Proceedings of the 5th International Workshop on Computational Kinematics, pp 51-60. Springer Verlag, 2009.

10. M. Coste, "A Simple Proof that Generic 3-RPR Manipulators Have Two Aspects", to appear in J. Mechanisms Robotics

11. V.I. ARNOL'D, S.M. GuSEǏn-ZADE, A.N. VARCHENKo, Singularities of differentiable maps Vol. I., Birkhäuser 1985.

12. E. FerRAND, "Des fronts d'onde en topologie", Gazette des Mathématiciens 123: 5-15, 2010.

13. D. PEdoE, "Thinking geometrically", Amer. Math. Monthly 77: 711-721, 1970.

14. D.S. Mitrinović, J.E. PeČARIĆ, "About the Neuberg-Pedoe and the Oppenheim inequalities", J. Math. Anal. Appl. 129(1): 196-210, 1988.

15. M. Coste, P. Wenger, D. Chablat, "Singular surfaces and cusps in symmetric planar 3RPR manipulators", Proc. of IEEE/RSJ International Conference on Intelligent Robots and Systems, San Francisco, 2011. 